\title{
A COMPLETION FUNCTOR FOR CAUCHY GROUPS
}

\author{
R. FRIC \\ D.C. KENT \\ Department of Pure and Applied Mathematics \\ Washington State University \\ Pullman, Washington 99164 \\ U.S.A.
}

(Received March 11, 1980)

ABSTRACT. A completion functor is constructed on the category of completely normal Cauchy groups and Cauchy-continuous homomorphisms. A competion functor is also obtained for a corresponding category of convergence groups.

KEY WORDS AND PHRASES. Completion functor, Cauchy group, pre-Cauchy group, completely normal Cauchy group, convergence group.

1980 MATHEMATICS SUBJECT CLASSIFICATION CODES: 54A20, 54E15, 18 A35

\section{INTRODUCTION.}

In [2], we developed a general functorial approach to Cauchy space completions. In this paper, we consider completions of groups equipped with compatible Cauchy structures. Such spaces have been studied by R.N. Ball [1], who used them to obtain certain completions of lattice ordered groups.

We shall use the definitions, notation, and terminology of [2]. However the modification functor approach to constructing completion functors used in 
[2] is not employed here. Although at least some of our results could be obtained via that method, it is found to be less convenient due, in part, to the fact that Wyler's completion does not preserve the compatibility between group and Cauchy structures.

Let PCHG be the category whose objects are groups equipped with Cauchy structures relative to which the group operations are Cauchy continuous (such groups are said to be pre-Cauchy), and whose morphisms are Cauchy-continuous homomorphisms. All Cauchy space categories considered here will be subcategories of PCHG. For convenience, we shall use the term "subcategory" to mean "full subcategory", so that subcategories are completely determined by their objects. In applying the definitions of such terms in [2] as "map", "completion", etc. to our present setting it is, of course, necessary to replace "Cauchy-continuous function" by "Cauchy-continuous homomorphism", etc.

Throughout this paper, $X$ will represent a group with multiplicative operation and identity element $e ; F(X)$ will denote the set of all filters on $X$, and $\dot{x}$ will denote the fixed ultrafilter determined by $x \in X$. Group operations are applied to subsets and filters in the obvious way. In particular, for $z, \& \in F(X), 3^{-1}=\left\{F^{-1}: F \in Z\right\}$ and $z \&$ is the filter generated by $\{F G: F \in Z, G \in \&\}$.

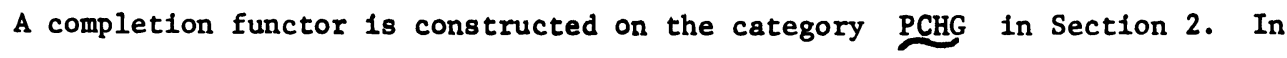
Section 3, we consider the subcategory CHG of PCHG consisting of Cauchy groups, and show that the completely normal Cauchy groups form a completion subcategory of CHG. Whether a completion functor exists on all of CHG remains an unsolved problem at this time. Section 4 examines the relationships between Cauchy groups and convergence groups. The results of Section 3 are used to construct a completion functor on the category of completely normal convergence groups and continuous homomorphisms. 


\section{PRE-CAUCHY GROUPS}

A pre-Cauchy group is a pair $(X, C)$, where $X$ is a group and $C$ a set of filters on $X$ satisfying the following conditions:

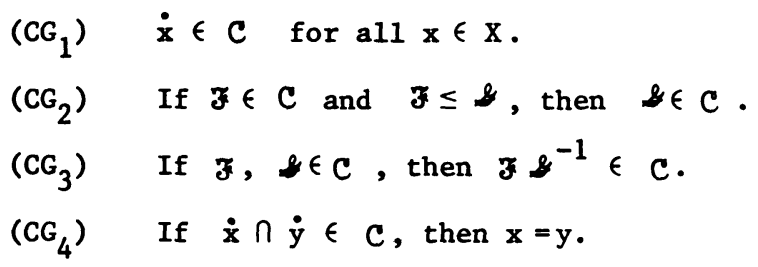

Note that if $(x, C)$ is a pre-Cauchy group, then $C$ is closed under the formation of inverses and products of filters. It is easy to see that if $x$ is a separated (Hausdorff) topological group and $C$ is the set of all Cauchy filters, then $(X, C)$ is a pre-Cauchy group.

PROPOSITION 2.1 If $(\mathrm{X}, \mathrm{C})$ is a pre-Cauchy group, then $\mathrm{C}$ is a Cauchy structure on $\mathrm{X}$.

PROOF. The first two Cauchy space axioms (see [2]) are covered by (CG $)$ and $\left(\mathrm{CG}_{2}\right)$. If $\mathrm{z}$ and $\&$ are nondisjoint filters in $\mathrm{C}$, then $\mathrm{z} \& \geq \mathrm{z}^{-1} \&$, and therefore $\cap \& \in C$; this establishes the third Cauchy space axiom.

The Cauchy structure associated with a pre-Cauchy group is called a "group Cauchy structure" in [1]. This should not be confused with our term "Cauchy group structure", by which we shall mean the Cauchy structure associated with a Cauchy group (see Section 3).

Starting with a pre-Cauchy group $(\mathrm{X}, \mathrm{C})$, let $\mathrm{x}^{*}$ be the set of Cauchy equivalence classes and $j: X \rightarrow x^{*}$ the canonical map. Given [3], [\&] $\epsilon x^{*}$, define $[\xi][\&]=[\xi \xi]$ and $[\xi]^{-1}=\left[\xi^{-1}\right]$.

As in [2] we associate with a Cauchy space (X,C) the convergence structure on $x$ defined by : $z \rightarrow x$ if $z \cap \dot{x} \in C$ (or, equivalently, if $z \in[\dot{x}]$ ). For pre-Cauchy groups, equivalence of Cauchy filters is characterized in terms of convergence in the next proposition. 
PROPOSITION 2.2 Let $(\mathrm{X}, \mathrm{C})$ be a pre-Cauchy group and $z, \& \in \mathrm{C}$. Then $z \cap \& \in C$ iff $z^{-1} \rightarrow$ e.

PROOF. Let $\sharp=z \cap \&$. Then $z^{-1} \geq z^{-1} \in C$, and $\dot{e} \geq \sharp z^{-1}$ implies

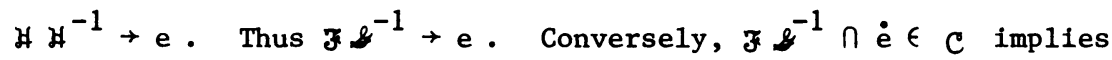
$z \cap \& \geq\left(z^{-1} \cap \dot{e}\right) \& \in C$.

PROPOSITION 2.3 If $(\mathrm{X}, \mathrm{C})$ is a pre-Cauchy group, then $\mathrm{x}^{*}$, equipped with the operations defined above, is a group, and $\mathrm{j}: \mathrm{X} \rightarrow \mathrm{x}^{*}$ is an isomorphic embedding.

PROOF. We shall verify only that the multiplicative operation on $x^{*}$ is well-defined. Let $\left[z_{1}\right]=\left[z_{2}\right]$ and $\left[\xi_{1}\right]=\left[\&_{2}\right]$. By Proposition 2.2, ${ }_{1} z_{2}{ }^{-1} \rightarrow \mathrm{e}$ and $\&_{1} \&_{2}^{-1} \rightarrow \mathrm{e}$, and it suffices to show $\left(z_{1} \xi_{1}\right)\left(z_{2} \xi_{2}\right)^{-1} \rightarrow \mathrm{e}$. Note that $z_{1} z_{2}^{-1} \geq z_{1}\left(\&_{1} \&_{2}^{-1} \cap \dot{e}\right) z_{2}^{-1} \in C$. If $\sharp=z_{1}\left(\&_{1} \&_{2}^{-1} \cap \dot{e}\right) z_{2}^{-1}$ and $k=z_{1} z_{2}^{-1} \cap \dot{e}$, then $\sharp \cap x \in C$, and $\left(z_{1} \xi_{1}\right)\left(z_{2} \xi_{2}\right)^{-1} \cap \dot{e} \geq \sharp \cap k$, which implies $\left(F_{1} \&_{1}\right)\left(z_{2} \&_{2}\right)^{-1} \rightarrow$ e.

Let $(X, C)$ be a pre-Cauchy group. For each free filter $\exists \in C$, define the filter $\Phi$ on $X^{*}$ as follows: $\Phi=j(\xi) \cap[\dot{\xi}]$. Filters of the form $\Phi_{Z}$, for ${ }^{3}$ a free filter in $C$, and also all fixed ultrafilters on $x^{*}$, will be called subbasic filters on $x^{*}$. Any filter on $x^{*}$ obtained by taking a finite product of subbasic filters will be called a basic filter. Let $\mathrm{C}^{*} \subseteq \mathrm{F}\left(\mathrm{X}^{*}\right)$ be the set of filters defined by:

$$
C^{*}=\left\{\psi \in F\left(X^{*}\right): \psi \geq \wedge \text { for some basic filter } \wedge\right\} \text {. }
$$

LEMMA 2.4 A basic filter on $x^{*}$ is either a fixed ultrafilter, or else a filter of the form $\psi \cap \dot{\alpha}$, where $\psi$ is a free filter and $\alpha \in \mathrm{x}^{*}$.

PROOF. A basic filter $\wedge$ on $x^{*}$ can be written as a product $\wedge_{1} \wedge_{2} \cdots \wedge_{n}$, where each $\wedge_{i}$ is of the form $\Phi_{i} \cap\left[\dot{\vec{z}}_{i}\right]$, and $\Phi_{i}=\left[\dot{H}_{i}\right] j\left(\&_{i}\right)\left[\dot{x}_{i}\right]$, where

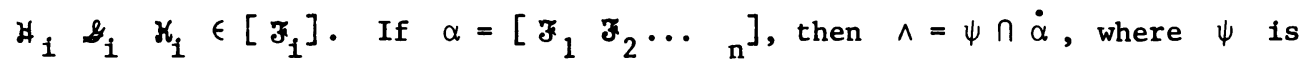
the intersection of translations of free filters, and hence a free filter. 
THEOREM 2.5. If $(X, C)$ is a pre-Cauchy group, then $\left(\left(X^{*}, C^{*}\right), j\right)$ is a strict pre-Cauchy group completion of (X, C ).

PROOF. The axioms $\left(\mathrm{CG}_{1}\right),\left(\mathrm{CG}_{2}\right)$, and $\left(\mathrm{CG}_{3}\right)$ follow immediately from the construction of $C^{*}$. Axiom $\left(\mathrm{CG}_{4}\right)$ follows easily from Lemma 2.4, and so $\left(\mathrm{x}^{*}, \mathrm{C}^{*}\right)$ is a pre-Cauchy group. It also follows from Lemma 2.4 that $\left(\mathrm{X}^{*}, \mathrm{C}^{*}\right)$ is complete, since each member of $C^{*}$ is finer than a basic filter of the form $\psi \cap \dot{\alpha}$ and hence convergent to $\alpha$. We know already that $j:(x, c) \rightarrow\left(x^{*}, c^{*}\right)$ is an isomorphic embedding, and this map is clearly Cauchy-continuous. Cauchycontinuity of $j^{-1}$ follows because $j^{-1}$ is an isomorphism and $j^{-1}(\wedge) \in C$ whenever $\wedge$ is a subbasic filter containing $f(x)$. Therefore $\left(\left(x^{*}, c^{*}\right), j\right)$ is a pre-Cauchy group completion of (X,C ).

To show that this completion is strict, let $\psi \rightarrow \alpha$ in $\mathrm{x}^{\star}$. Then there is $\wedge \in C^{*}$ such that $\psi \geq \wedge$. Assume that $\wedge=\wedge_{1} \wedge_{2} \ldots \wedge_{n}$, where each $\Lambda_{1}=\left[\dot{z}_{i}\right] j\left(\&_{i}\right)\left[\dot{x}_{j}\right]$. Then $c 1 \psi \geq c 1 \wedge \geq c 1 j\left(\psi_{1} \&_{1} \kappa_{1} \ldots z_{n} \& \psi_{n}\right)$, and the conclusion follows.

THEOREM 2.6. If $\mathrm{f}:(\mathrm{X}, \mathrm{C}) \rightarrow\left(\mathrm{X}_{1}, \mathrm{C}_{1}\right)$ is a Cauchy-continuous homomorphism, and $\left(\mathrm{x}_{1}, \mathrm{C}_{1}\right)$ is a complete pre-Cauchy group, then there is a unique Cauchycontinuous homomorphism $\overline{\mathrm{f}}$ which makes the following diagram commute:

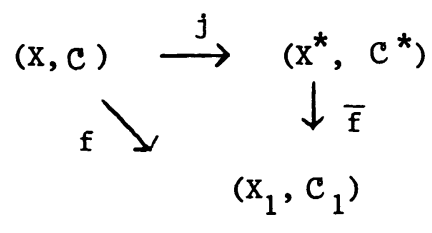

PROOF. For each $z \in C$, define $\bar{f}([z])=y$ iff $f(z) \rightarrow y$ in $x_{1}$; clearly $\bar{f}$ is well-defined. Let $\bar{f}([\mathfrak{x}]) \bar{f}([\&])=y z$, where $f(z)+y$, $f(\xi) \rightarrow z$. Then $f(\xi) f(\xi)=f(\xi \&)+y z$, and so $\bar{f}([\xi \&])=\bar{f}([\xi][\&])=y z$, which implies that $\bar{f}$ is a homomorphism. If $\phi$ is a subbasic filter and $\phi \rightarrow \alpha$ in $x^{*}$, then it is clear that $\bar{f}(\phi) \rightarrow \bar{f}(\alpha)$ in $x_{1}$; the fact that $\bar{f}$ is 
Cauchy-continuous follows from the fact that $\overline{\mathrm{f}}$ is a homomorphism. The uniqueness of $\bar{f}$ follows because all spaces involved are Hausdorff.

Let PCHG be the category of pre-Cauchy groups and Cauchy-continuous homomorphisms, and let $\mathrm{PCHG}^{*}$ be the subcategory of complete objects in $\mathrm{PCG}$. For objects $(X, C) \in$ PCHG, define $K(X, C)=\left(X^{*}, C^{*}\right)$. If $\mathrm{f}:\left(\mathrm{X}_{1}, \mathrm{C}_{1}\right) \rightarrow\left(\mathrm{X}_{2}, \mathrm{C}_{2}\right)$ is a morphism in $\mathrm{PCHG}$, define $\mathrm{Kf}:\left(\mathrm{x}_{1}{ }^{*}, \mathrm{C}_{1}^{*}\right) \rightarrow\left(\mathrm{x}_{2}^{*}, \mathrm{C}_{2}{ }^{*}\right)$ be the unique extension morphism whose existence is guaranteed by Theorem 2.6. Then $\mathrm{K}: \mathrm{PCHG}^{\mathrm{PCHG}}{ }^{*}$ is a covariant functor. If, in the notation of [2], we identify $i_{k}$ with the embedding $j: x \rightarrow x^{*}$, then the following corollary is an immediate consequence of Theorem 2.6.

COROLLARY 2.7. $\mathrm{K}$ is a strict completion functor on PCHG.

\section{CAUCHY GROUPS.}

A pre-Cauchy group (X,C) is said to be a Cauchy group if the following condition is satisfied: $\left(\mathrm{CG}_{5}\right) \mathrm{z}^{-1} \in \mathrm{C}$ and $\mathrm{z}^{-1} \mathrm{z} \in \mathrm{C} \Rightarrow \mathrm{z} \in \mathrm{C}$.

It is natural to ask whether $\left(\mathrm{X}^{*}, \mathrm{C}^{*}\right)$ is a Cauchy group whenever (X,C) is a Cauchy group. The answer is not known in general, but the answer is yes for a certain class of Cauchy groups which we shall now discuss.

LEMMA 3.1. The following conditions on a Cauchy group (X,C ) are equivalent.

$\left(\mathrm{CN}_{1}\right)$ If $₹, \& \in \mathrm{C}$ are non-convergent, then $\& 3^{-1}$ is non-convergent. $\left(\mathrm{CN}_{2}\right)$ If $\quad \mathrm{C} \in$ and $\mathrm{x} \in \mathrm{X}$, then $\mathrm{J}^{-1} \dot{\mathrm{x}} \mathrm{z}$ is convergent. $\left(\mathrm{CN}_{3}\right) \quad j(\mathrm{X})$ is a normal subgroup of $\mathrm{X}^{*}$. PROOF. $\left(\mathrm{CN}_{1}\right) \Rightarrow\left(\mathrm{CN}_{2}\right)$. If $\in C$ and $3^{-1} \dot{x}$ is non-convergent, then $z$ and $\&$ are both non-convergent members of $C$, where $\&=z^{-1} \dot{x}$. But $\dot{x} \geq z^{-1} \dot{x} z^{-1}=z^{-1}$, which implies that $z^{-1} \rightarrow x$. This contradicts the assertion of $\left(\mathrm{CN}_{1}\right)$ that $\xi^{-1}$ must be non-convergent.

$\left(\mathrm{CN}_{2}\right) \Rightarrow\left(\mathrm{CN}_{3}\right)$. We shall show that $\alpha^{-1} j(X) \alpha=j(X)$ for all $\alpha \in X^{*}-j(X)$. 
If $z \in \alpha \in \mathrm{X}^{*}$, and $\mathrm{x} \in \mathrm{X}$, then $\left[\mathrm{z}^{-1} \dot{\mathrm{x}}\right]=\alpha^{-1} \mathrm{j}(\mathrm{x}) \alpha \in \mathrm{j}(\mathrm{X})$ by $\left(\mathrm{CN}_{2}\right)$. Conversely, let $x \in X, \beta=\alpha j(x) \alpha^{-1}$. If $\in \alpha$, then $\dot{x} z^{-1} \rightarrow y$ for some $y \in X$ : thus $y=j^{-1}(\beta)$, and $j(x)=\alpha^{-1} j(y) \alpha \in \alpha^{-1} j(x) \alpha$.

$\left(\mathrm{CN}_{3}\right) \Rightarrow\left(\mathrm{CN}_{1}\right)$. Let $\&$ be non-convergent filters in $\mathrm{C}$, and let $\alpha=[\mathrm{z}], \beta=[\&]$. If $z^{\prime} \mathfrak{z}^{-1}$ is convergent, then $\alpha \beta \alpha^{-1}=j(x)$ for some $\mathrm{x} \in \mathrm{X}$, and $\beta=\alpha^{-1} \mathrm{j}(\mathrm{x}) \alpha \in \mathrm{j}(\mathrm{X})$ by $\mathrm{CN}_{3}$. This implies that \& is convergent, and this contradiction completes the proof.

A Cauchy group ( $\mathrm{x}, \mathrm{C})$ which satisfies any of the three equivalent conditions of Lemma 3.1 is said to be completely normal. Note that all complete Cauchy groups and all Abelian Cauchy groups are completely normal.

THEOREM 3.2. If $(X, C)$ is completely normal, then $\left(X^{*}, C^{*}\right)$ is a Cauchy group.

Proof. We need to show that $\left(\mathrm{X}^{*}, \mathrm{C}^{*}\right)$ satisfies $\left(\mathrm{CG}_{5}\right)$. Assume that $\psi \psi^{-1} \in \mathrm{C}^{*}$; then there are subbasic filters $\wedge_{1}, \ldots, \wedge_{\mathrm{n}}$ such that $\psi \psi^{-1} \geq \wedge_{1} \wedge_{2} \cdots \wedge_{\mathrm{n}}$. Recalling the form of the subbasic filters, we can argue that any finite product of subbasic filters is also expressible in the form $\prod_{i=1}^{m} \Phi_{i}$, where each $\Phi_{i}=\dot{\beta}_{i 1} j\left(\xi_{i 1}\right) \dot{\beta}_{i 2} j\left(\xi_{i 2}\right) \ldots \dot{\beta}_{i k i} j\left(\xi_{i k_{i}}\right)$, where $\beta_{i j} \in x^{*}$ and $\&_{i j} \in C$. If $(X, C)$ is completely normal, then the expression for $\Phi_{1}$ can be reduced to one of the form $\Phi_{i}=\dot{\beta}_{i} j\left(\xi_{i}\right) \dot{\gamma}_{i}$, where $\beta_{i}, \gamma_{i} \in x^{*}$ - and $\xi_{i} \in C$. Thus, we shall assume that $\psi \psi^{-1} \geq \prod_{i=1}^{m} \dot{\beta}_{i} j\left(\xi_{i}\right) \dot{\gamma}_{i}$, where $\beta_{i}, \gamma_{i}$, and $\&_{i}$ are as previously specified.

For each $1,1 \leq i \leq m$, choose $G_{i} \in \&_{i}$. Then there is $A \in \psi$ such that $A A^{-1} \subseteq \bigcup_{i=1}^{m} B_{i} j\left(G_{i}\right) \gamma_{i}$. If $\delta \in A$, then $A \delta^{-1} \subseteq \bigcup_{i=1}^{m} \beta_{i} j\left(G_{i}\right) \gamma_{i}$. If $A^{\prime} \subseteq A$ and $A^{\prime} \in \psi$, then for each $i$ there is $G_{i}{ }^{\prime} \subseteq G_{i}$ (but $G_{i}^{\prime}$ is not necessarily in $\&_{i}$ ) such that $A^{\prime} \delta^{-1} \subseteq \bigcup_{i=1}^{m} B_{i} G_{i}^{\prime} \gamma_{i}$. From these observations, it follows that there is at least one index $\ell, 1 \leq \ell \leq \mathrm{m}$, for which there exists a filter $z_{\ell}$ (not necessarily in $C$ ) such that $\psi \dot{\delta}^{-1}$ and $\dot{\beta}_{\ell} j\left(H_{\ell}\right) \dot{\gamma}_{\ell}$ are filters 
which do not contain disjoint sets.

Let $\psi_{1}=\dot{\beta}_{\ell} j\left(z_{\ell}\right) \dot{\gamma}_{\ell} \dot{\delta}$. Then $j\left(z_{\ell} l_{\ell}^{-1}\right)=\dot{\beta}_{\ell}^{-1} \psi_{1} \psi_{1}^{-1} \dot{\beta}_{\ell} \geq \dot{\beta}_{\ell}^{-1} \psi \psi^{-1} \dot{\beta}_{\ell}$. This implies that $j\left(\sharp_{\ell} \ell_{\ell}^{-1}\right) \in C^{*}$, which implies $\sharp_{\ell} \|_{\ell}^{-1} \in C$, which, by $\left(\mathrm{CG}_{5}\right)$, implies that $z_{\ell} \in \mathrm{C}$. From this, it follows that $\psi_{1} \in \mathrm{C}^{*}$. Thus $\psi \psi_{1}^{-1} \geq \wedge_{1} \wedge_{2} \cdots \wedge_{n}$ implies $\psi \geq \wedge_{1} \wedge_{2} \cdots \wedge_{n} \psi_{1}$; since each of the filters in the latter product is in $\mathrm{C}^{*}, \psi \in \mathrm{C}^{*}$, and the proof is complete.

Let CNCHG be the subcategory of PCHG determined by the completely normal Cauchy groups, and let $\mathrm{CNCHG}^{*}$ denote the subcategory of complete objects in CNCHG. It follows from Theorem 3.2 that the functor $K$ maps CNCHG into $\mathrm{CNCHG}^{*}$. Combining this fact with Corollary 2.7 , and using the same symbol $\mathrm{K}$ for the restricted functor, we obtain

THEOREM 3.3. $\mathrm{K}$ is a completion functor on the category CNCHG of completely normal Cauchy groups.

In other words, the completely normal Cauchy groups form a completion subcategory of the pre-Cauchy groups. The completion by $K$ of an Abelian Cauchy group is clearly Abelian; thus we have also established

COROLLARY 3.4. $\mathrm{K}$ is a completion functor on the category of Abelian Cauchy groups.

\section{CONVERGENCE GROUPS.}

A convergence group $(X, q)$ is a pair consisting of group $X$ and a convergence structure $q$ relative to which the group operations are continuous; that is, $\mathrm{z}^{\mathrm{q}} \rightarrow \mathrm{x}$ and $\&^{q} \mathrm{y}$ implies $\mathrm{z}^{-1} \stackrel{\mathrm{q}}{\rightarrow} \mathrm{xy}^{-1}$. With each convergence group $(X, q)$ there is associated a natural Cauchy structure $C_{q}=\left\{z \in F(X): z^{-1} \stackrel{q}{\rightarrow} e\right.$ and $\left.z^{-1} z^{\rightarrow} e\right\}$. On the other hand, every preCauchy group (X, C) gives rise to a convergence structure ${ }^{q_{C}} \mathrm{C}$ on $\mathrm{X}$ defined

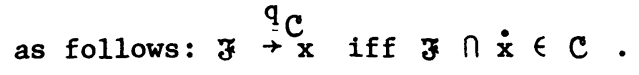

A convergence group $(X, q)$ is said to be strongly normal if $\mathfrak{F}^{-1} \stackrel{q}{\rightarrow} e$, 
$z^{-1} \& \stackrel{q}{\rightarrow} e$, and $\& \stackrel{q}{\rightarrow} e$ implies $z z^{-1} \stackrel{q}{\rightarrow}$ e. The definition of strong normality and statement (b) of the next proposition are due to R.N. Ball, [1].

PROPOSITION 4.1. (a) If $(X, C)$ is a pre-Cauchy group, then $\left(X, q_{C}\right)$ is a convergence group.

(b) If $(X, q)$ is a convergence group, then $\left(X, C_{q}\right)$ is a pre-Cauchy group iff $(X, q)$ is strongly normal.

(c) If $(X, q)$ is a convergence group and $\left(X, C_{q}\right)$ is a pre-Cauchy group, then $\left(\mathrm{X}, \mathrm{C}_{\mathrm{q}}\right)$ is a Cauchy group.

PROOF. (a) Let $\stackrel{{ }^{\mathrm{q}} \mathrm{C}}{\rightarrow} \mathrm{x}, \quad \stackrel{{ }^{\mathrm{q}} \mathrm{C}}{\rightarrow} \mathrm{y}$. Then $z^{-1} \stackrel{{ }^{\mathrm{q}}}{\rightarrow} \mathrm{C}_{\mathrm{x}} \mathrm{y}^{-1}$ because $z \cap \dot{x} \in C, \quad g^{-1} \cap \dot{y}^{-1} \in C$, and $z^{-1} \cap\left(\dot{x}^{-1}\right) \geq(z \cap \dot{x})\left(g^{-1} \cap \dot{y}^{-1}\right) \in C$.

(c) Obvious.

PROPOSITION 4.2. For any group $\mathrm{X}$, the relation $\mathrm{C} \leftrightarrow \mathrm{q}_{\mathrm{C}}$ is a one-to-one correspondence between Cauchy group structures and strongly normal convergence group structures on $\mathrm{X}$.

PROOF. If $(X, C)$ is a Cauchy group, then $\left(X, q_{C}\right)$ is a strongly normal convergence group by Proposition 4.1. If $C^{\prime}=C_{q_{C}}$, then $C^{\prime}$ is also a Cauchy group structure by Proposition 4.1 , and it remains to show that $\mathrm{C}=\mathrm{C}^{\prime}$. If $z_{C}$, then $z^{-1} \in C$ and $z^{-1} \in C$, and since $\dot{e}$ is finer than $z^{-1} z^{3}$ and $z^{-1}$, both of these product filters $q$-converge to $e$. Therefore, $z \in C^{\prime}$. Conversely, if $z \in C^{\prime}$, then $z^{-1} z^{q} \stackrel{C}{\rightarrow}$ and $z^{-1} \stackrel{{ }^{q}}{\rightarrow}$ e. Thus $z^{-1}$ and $z^{-1} z$ are both in $C$, and $z \in C$ follows by $\left(\mathrm{CG}_{5}\right)$.

It is obvious that Abelian convergence groups are strongly normal. Thus we obtain

COROLLARY 4.3. If $X$ is an Abelian group, then $C_{q} \leftrightarrow q$ is a one-to-one correspondence between Cauchy group structures and convergence group structures on $\mathrm{X}$. 
PROPOSITION 4.4. (a) If $\varphi:(\mathrm{X}, \mathrm{C}) \rightarrow\left(\mathrm{X}_{1}, \mathrm{C}_{1}\right)$ is a Cauchy-continuous homomorphism between pre-Cauchy groups, then $\varphi:\left(x_{,} q_{C}\right) \rightarrow\left(x_{1}, q_{C_{1}}\right)$ is continuous.

(b) If $\varphi:(X, q) \rightarrow\left(X_{1}, q_{1}\right)$ is a continuous homomorphism between strongly normal convergence groups, then $\varphi:\left(\mathrm{X}, \mathrm{C}_{\mathrm{q}}\right) \rightarrow\left(\mathrm{X}_{1}, \mathrm{C}_{\mathrm{q}_{1}}\right)$ is Cauchy-continuous.

(c) If $(X, C)$ is a complete Cauchy group, then $\left(X, q_{C}\right)$ is a complete convergence group.

(d) If $(X, q)$ is a complete convergence group, then $\left(X, C_{q}\right)$ is a complete Cauchy group.

PROOF. Proofs are given only for (b) and (c), the other parts being similar. (b) Let $z \in \mathbb{C}_{\mathrm{q}}$. Then $\mathfrak{z}^{-1} \stackrel{\mathrm{q}}{\rightarrow}$ e implies $\varphi\left(\mathrm{z}^{-1}\right)=\varphi(x) \varphi(x)^{-1} \stackrel{\mathrm{q}_{1}}{\rightarrow}$. Similarly, $\varphi(z)^{-1} \varphi(z) \stackrel{q_{1}}{\rightarrow} \mathrm{e}$, and so $\varphi(z) \in \mathrm{c}_{\mathrm{q}_{1}}$.

(c) Let $(\mathrm{X}, \mathrm{C})$ be a complete pre-Cauchy group, and let $\mathrm{z}^{-1} \stackrel{\mathrm{q}^{\mathrm{q}} \mathrm{C}}{\rightarrow}$ and $F^{-1}{ }_{\rightarrow}^{q} \mathrm{C}$. Then $z \in \mathrm{C}_{\mathrm{q}_{\mathrm{C}}}=\mathrm{C}$ as was shown in the proof of Proposition 4.2,

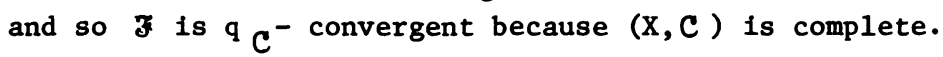

We define a convergence group $(X, q)$ to be completely normal if it is strongly normal and $\left(\mathrm{x}, \mathrm{C}_{\mathrm{q}}\right)$ is a completely normal Cauchy group. Note that all Abelian convergence groups and complete convergence groups are completely normal.

Let CNCG denote the category whose objects are completely normal convergence groups and whose morphisms are continuous homomorphisms: let $\mathrm{CNCG}^{*}$ denote the subcategory determined by the complete objects in CNCG. Define $\overline{\mathrm{K}}:$ CNCG $\rightarrow$ CNCG$^{*}$ by $\overline{\mathrm{K}}(\mathrm{X}, \mathrm{q})=\left(\mathrm{X}^{*}, \mathrm{q}^{*}\right)$, where $\mathrm{X}^{*}$ is the set of $\mathrm{C}_{\mathrm{q}}$-equivalence classes, and $q^{*}=q_{q^{*}}$; the fact that $\left(X^{*}, q^{*}\right) \in \operatorname{CNCG}^{*}$ is a consequence of Proposition 4.4. Using Proposition 4.4 along with the results of Section 3, we obtain the following.

THEOREM 4.5. $\overline{\mathrm{K}}$ is a completion functor on the category CNCG of completely normal convergence groups. In particular, $\bar{K}$ is a completion functor on the 
on the subcategory of CNCG consisting of all Abelian convergence groups.

The existence of a completion functor on the Abelian convergence group

category was established in [3] using methods and terminology based on [4].

\section{REFERENCES}

[1] R.N. Ball, "Convergence and Cauchy Structures on Lattice Ordered Groups", Trans. Amer. Math. Soc. (to appear).

[2] R. Frǐ and D.C. Kent, "Completion Functors for Cauchy Spaces", International J. Math. \& Math Sci. 2(1979) 589-604.

[3] R. Fric and D.C. Kent, "Completion of Pseudo-Topological Groups", Math. Nachr. (to appear).

[4] S. Gähler, and G. Kneis, "Completion of Pseudo-Topological Vector Spaces", Math. Nachr. 75(1976) 185-206. 


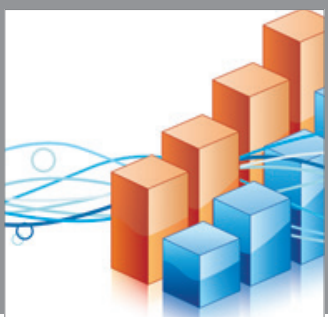

Advances in

Operations Research

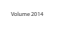

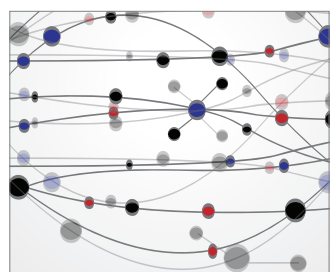

\section{The Scientific} World Journal
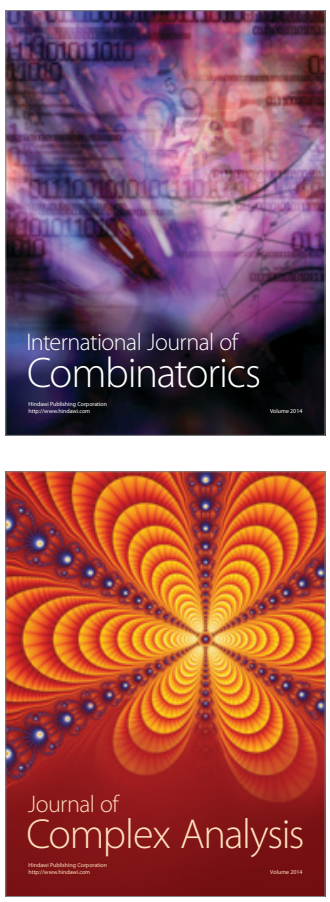

International Journal of

Mathematics and

Mathematical

Sciences
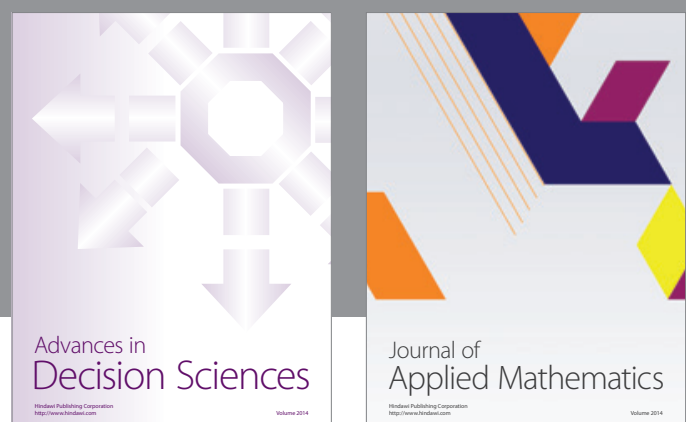

Journal of

Applied Mathematics
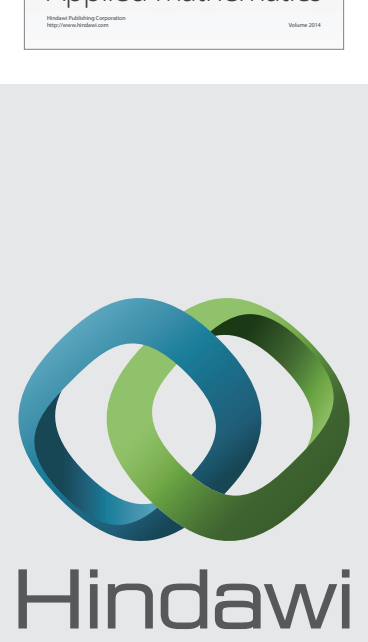

Submit your manuscripts at http://www.hindawi.com
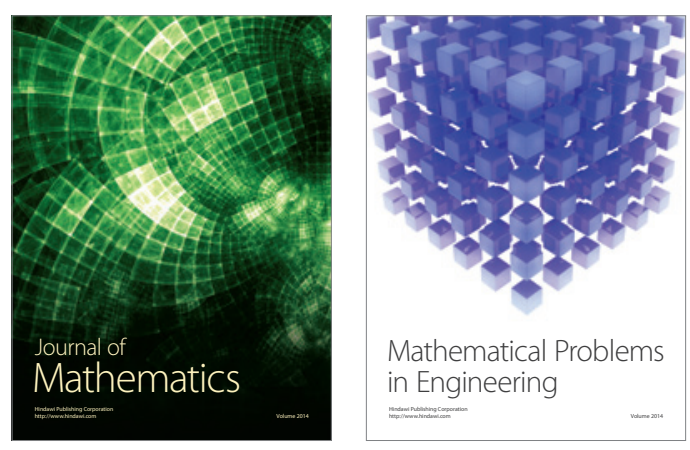

Mathematical Problems in Engineering
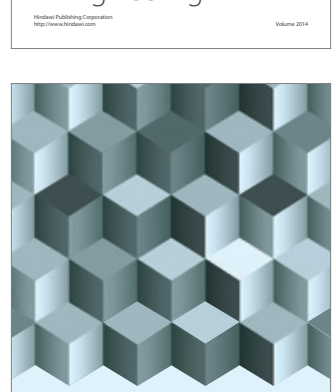

Journal of

Function Spaces
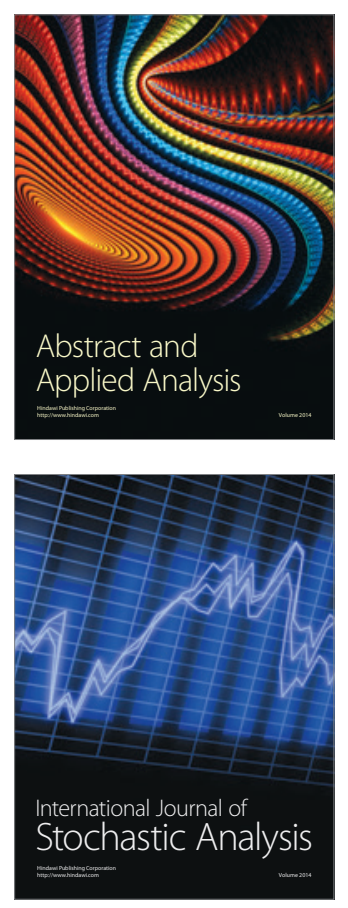

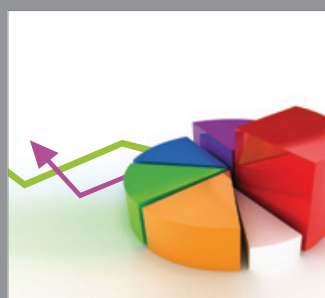

ournal of

Probability and Statistics

Promensencen
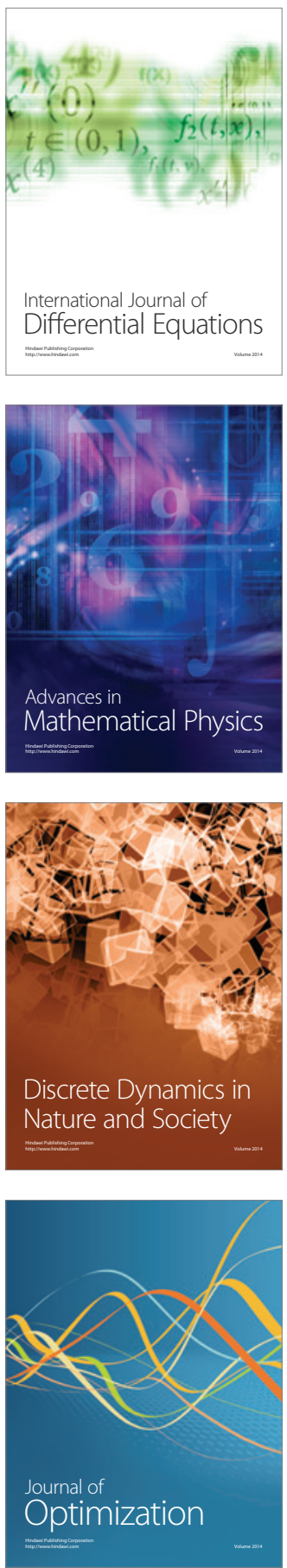